\title{
Efficacy and tolerability of the fixed combination of brinzolamide $1 \%$ and timolol $0.5 \%$ in daily practice
}

This article was published in the following Dove Press journal:

Clinical Ophthalmology

| March 201 |

Number of times this article has been viewed

\author{
Ines Lanzl' \\ Thomas Raber ${ }^{2}$ \\ 'Eye Department of the Technical \\ University, Munich, Germany; ${ }^{2}$ Alcon \\ Pharma GmbH, Freiburg, Germany
}

Introduction: The purpose of this study was to document the efficacy and tolerability of the new fixed-combination (FC) brinzolamide $1 \% /$ timolol $0.5 \%$ as used in daily practice throughout Germany.

Methods: An open-label, multicenter, observational study of patients was performed that evaluated the transition from prior medication to brinzolamide/timolol FC for the reduction of intraocular pressure (IOP). Ophthalmologists measured IOP at baseline and 4-6 weeks after transition and assessed their satisfaction with brinzolamide/timolol FC. Patients assessed the tolerability of both their previous and new regimens, judged their satisfaction with brinzolamide/timolol, and reported their preference between their previous and new regimens. Patients transitioning from other FC products to brinzolamide/timolol FC were analyzed separately.

Results: Data from 14,025 patients from 1161 centers were analyzed. Four to 6 weeks after transition to brinzolamide/timolol, patients experienced a mean IOP decrease of $3.9 \pm 4.3 \mathrm{~mm} \mathrm{Hg}$ $(P<0.0001)$. All of the predefined patient subgroups (grouped by previous therapy) demonstrated a significant mean IOP reduction following transition to brinzolamide/timolol $(P<0.0001)$. Patients judged brinzolamide/timolol tolerability more positively than they did their previous therapies ( $87.2 \%$ vs $53.7 \%$ favorable assessments) and reported a high satisfaction rating with brinzolamide/timolol (93.4\%). Brinzolamide/timolol was preferred over previous therapy at a ratio of almost 9:1. Patients who transitioned from dorzolamide/timolol to brinzolamide/timolol $(\mathrm{n}=2937)$ demonstrated a significant decrease in mean IOP $(P<0.0001)$, rated brinzolamide/timolol more tolerable than dorzolamide/timolol ( $88.9 \%$ vs $28.9 \%)$, and preferred brinzolamide/timolol at a ratio of more than 9:1. Patients who transitioned from brimonidine/timolol $(\mathrm{n}=209)$ demonstrated a significant decrease in mean IOP $(P<0.0001)$, rated brinzolamide/timolol more tolerable ( $86.5 \%$ vs $32.1 \%)$, and preferred brinzolamide/timolol at a ratio of 11.5:1.

Conclusions: The FC brinzolamide 1\%/timolol $0.5 \%$ produced better IOP control than all previous therapies analyzed and demonstrated favorable tolerability and a high satisfaction rating, resulting in a strong patient preference for brinzolamide/timolol over previous therapies.

Keywords: brinzolamide, fixed combination, glaucoma, intraocular pressure, timolol

\section{Introduction}

Several different classes of drugs are available for the reduction of elevated intraocular pressure (IOP) associated with open-angle glaucoma or ocular hypertension, including $\beta$-blockers, carbonic anhydrase inhibitors (CAIs), prostaglandin analogs (PGAs), and $\alpha-2$ agonists. Many patients, however, experience insufficient IOP reduction with 
single-agent therapy; in these cases, patients will transition to a two-drug regimen, which can be two individual agents or a fixed-combination (FC) product.

All of the currently available FC products combine the $\beta$-blocker timolol with another drug from a different class of IOP-lowering agents. The first FC product made available in 1998 combined the CAI dorzolamide $2 \%$ with timolol $0.5 \%\left(\right.$ Cosopt $^{\circledR}$, Merck \& Co., Inc., Whitehouse Station, NJ, USA). Since then, other FC products have reached the market, including the PGA-containing products latanoprost 0.005\%/timolol 0.5\% (Xalacom ${ }^{\circledR}$, Pfizer Inc., New York, NY, USA), travoprost 0.004\%/timolol 0.5\% (DuoTrav ${ }^{\circledR}$, Alcon Laboratories, Inc., Fort Worth, TX, USA), bimatoprost $0.03 \% /$ timolol $0.5 \%$ (Ganfort ${ }^{\circledR}$, Allergan Inc., Irvine, CA, USA), and the $\alpha-2$ agonist-containing brimonidine $0.2 \% /$ timolol $0.5 \%$ (Combigan $^{\circledR}$, Allergan Inc.). Most recently, FC brinzolamide 1\%/timolol 0.5\% (Azarga ${ }^{\circledR}$, Alcon Pharma GmbH, Puurs, Belgium) received regulatory approval by the European Medicines Agency in November 2008 for the treatment of patients for whom monotherapy provides insufficient IOP reduction. ${ }^{1}$

As a result of this approval, the German Medicines Act (AMG) requires that the pharmaceutical marketing authorization holder (Alcon Pharma) gathers data related to the use of Azarga ${ }^{\circledR}$ after its introduction to the market. ${ }^{2}$ A prospective, observational trial, in which the medication is prescribed in a clinical practice, can provide data from the actual use of this product. Thus, the goal of the current noninterventional study was to document the efficacy and tolerability of the new FC brinzolamide $1 \% /$ timolol $0.5 \%$ to comply with this requirement by the $\mathrm{AMG}$.

\section{Methods}

This was an open-label, multicenter, observational study of patients who were treated with brinzolamide/timolol FC for the reduction of IOP. Centers and patients were located throughout Germany to ensure adequate and representative samples for the subgroup analyses. All therapy decisions, including the decision to transition to brinzolamide/timolol, were made solely at the treating ophthalmologist's discretion. Inclusion and exclusion criteria were in accordance with the Azarga ${ }^{\circledR}$ Summary of Product Characteristics. ${ }^{1}$ All patients meeting these criteria who were transitioned to brinzolamide/ timolol from January 2010 to July 2010 were included. All patients must have had IOP data at both the baseline visit and the Week 4-6 visit.

The following data were collected by the ophthalmologists using standardized data sheets: patient initials, gender, age, glaucoma type, explanatory notes for the transition to brinzolamide/timolol, baseline IOP, and IOP at the Week 4-6 visit. IOP was measured at each site using the method that was standard for that clinical practice. Patients assessed both the tolerability of their previous IOP-lowering regimen and their brinzolamide/timolol regimen using the descriptors "very good", "good", "moderate", and "not satisfactory", and they reported their preference between their previous and new regimens. Both patients and ophthalmologists assessed their satisfaction with brinzolamide/timolol using the descriptors "excellent", "very good", "good", "satisfactory", and "not satisfactory".

A predefined subgroup analysis based on previous and new therapies was conducted in which change in IOP from the baseline visit to the Week 4-6 visit was calculated for patients who had been taking one of the following IOPlowering regimens and then transitioned to brinzolamide/ timolol FC: no previous therapy, timolol $0.5 \%$, brinzolamide $1 \%$, dorzolamide $2 \%$, FC dorzolamide $2 \%$ /timolol $0.5 \%$, PGA (bimatoprost $0.03 \%$, latanoprost $0.005 \%$, tafluprost $0.0015 \%$, or travoprost $0.004 \%$ ), FC PGA/timolol $0.5 \%$, FC brimonidine $0.2 \% /$ timolol $0.5 \%$, brinzolamide $1 \%+$ timolol $0.5 \%$, dorzolamide $2 \%+$ timolol $0.5 \%$, or PGA + timolol $0.5 \%$. Change in IOP from the baseline visit to the Week 4-6 visit was also assessed for patients who had been taking one of the following combination IOP-lowering regimens and then transitioned to PGA + brinzolamide/timolol: PGA + brinzolamide, PGA + dorzolamide, or PGA + dorzolamide/timolol.

Statistical analysis was performed by the contract research organization MedPharmTec ${ }^{\circledR}$ Services (Munich, Germany). Descriptive statistics were analyzed using frequency, median, mean, standard deviation, range, and quartile, depending on the parameter. IOP was analyzed as patient individual deviation analysis. The study was reported to the German Federal Institute for Drug and Medical Devices (BfArM).

\section{Results}

Data from 14,025 patients from 1161 centers were analyzed in the study ( $\mathrm{n}=14,021$ evaluable patients). The median age of these patients was 69 years and 58\% were female. Most patients were diagnosed with either primary open-angle glaucoma $(80.1 \%)$ or ocular hypertension $(8.1 \%)$ (Table 1$)$. The reasons for the transition in therapy to brinzolamide/ timolol FC varied, with some patients having more than one reason, but the large majority were transitioned due to either an insufficient reduction in IOP with previous therapy $(54.0 \%)$ or intolerance to previous therapy $(28.9 \%)$. 
Table I Patient demographics $(n=14,025)$

\begin{tabular}{ll}
\hline Demographic & $\begin{array}{l}\text { Percentage } \\
\text { of patients }\end{array}$ \\
\hline Gender & \\
Male & 42.0 \\
Female & 58.0 \\
Diagnosis & \\
Primary open-angle glaucoma & 80.1 \\
Ocular hypertension & 8.1 \\
Pseudoexfoliative glaucoma & 3.7 \\
Other & 8.1 \\
Reasons for transition to brinzolamide/timolol & \\
(multiple answers may apply) & \\
Insufficient intraocular pressure lowering under & 54.0 \\
previous therapy & \\
Intolerance to previous therapy & 28.9 \\
Progressive disease & 15.8 \\
Cost reduction & 14.7 \\
Vascular aspects & 8.7 \\
Insufficient patient compliance with previous therapy & 7.6 \\
\hline
\end{tabular}

\section{Efficacy}

The mean IOP at baseline for these patients was $20.7 \pm 5.0 \mathrm{~mm}$ Hg. After 4-6 weeks on brinzolamide/timolol, these patients had a mean IOP of $16.8 \pm 3.6 \mathrm{~mm} \mathrm{Hg}$ and a mean IOP decrease of $3.9 \pm 4.3 \mathrm{~mm} \mathrm{Hg}(18.8 \%)$ from baseline $(P<0.0001)$. A predefined prospective subgroup analysis of patients using one of 14 different IOP-lowering regimens comprised 10,497 patients, representing $75 \%$ of the total patient population (Table 2). Most of the remaining 25\% of patients not included in this analysis had been treated with $\alpha-2$ agonists, parasympathomimetics, $\beta$-blockers other than timolol, or other combinations (fixed or unfixed). All subgroups demonstrated a reduction of mean IOP after transitioning to brinzolamide/timolol, whether alone or in combination with a PGA. Figure 1 illustrates the magnitude of the change in mean IOP from baseline to the Week 4-6 visit, ranging from $1.9 \mathrm{~mm} \mathrm{Hg}$ mean reduction for those patients previously on a PGA + dorzolamide/timolol who transitioned to a PGA + brinzolamide/ timolol to a $4.8 \mathrm{~mm} \mathrm{Hg}$ reduction for patients previously on timolol alone who transitioned to brinzolamide/ timolol FC. Less than $4 \%$ of patients were given brinzolamide/ timolol FC as an off-label, first-line therapy. As would be expected of an untreated population, they experienced the largest mean reduction in IOP $(9.0 \mathrm{~mm} \mathrm{Hg} ; 35 \%)$ of all subgroups. All of the patient subgroups analyzed had a statistically significant reduction in IOP following transition to brinzolamide/timolol $(P<0.0001)$.

\section{Tolerability}

When asked about the tolerability of brinzolamide/timolol, $87.2 \%$ of patients judged it positively, with $35.7 \%$ reporting it to be very good and $51.5 \%$ reporting it to be good. Compared with patients' evaluation of their previous therapy, this represented an increase of positive tolerability assessments of 33.5\% (53.7\% very good or good with previous therapy vs $87.2 \%$ very good or good with brinzolamide/timolol therapy).

\section{Satisfaction}

Overall satisfaction with brinzolamide/timolol was similar between patient and investigator assessments, with $87.5 \%$

Table 2 Efficacy of study treatment for predefined patient subgroups $(n=10,497)$

\begin{tabular}{|c|c|c|c|c|}
\hline Previous therapy & New therapy & $\mathbf{n}$ & $\begin{array}{l}\text { Mean IOP at } \\
\text { baseline ( } \mathrm{mm} \mathrm{Hg})\end{array}$ & $\begin{array}{l}\text { Mean IOP } 4-6 \text { weeks } \\
\text { after transition }(\mathrm{mm} \mathrm{Hg})\end{array}$ \\
\hline None $^{a}$ & Brinzolamide I\%/timolol 0.5\% & 435 & $25.7 \pm 6.3$ & $16.7 \pm 3.5$ \\
\hline Timolol $0.5 \%$ & Brinzolamide $1 \% /$ timolol $0.5 \%$ & 2446 & $21.9 \pm 3.9$ & $17.1 \pm 3.0$ \\
\hline Brinzolamide $1 \%$ & Brinzolamide $1 \% /$ timolol $0.5 \%$ & 933 & $20.9 \pm 4.1$ & $16.4 \pm 3.2$ \\
\hline Dorzolamide $2 \%$ & Brinzolamide $1 \% /$ timolol $0.5 \%$ & 458 & $21.3 \pm 4.7$ & $16.8 \pm 3.7$ \\
\hline Prostaglandin analog (PGA) ${ }^{b}$ & Brinzolamide $1 \% /$ timolol $0.5 \%$ & 1015 & $20.7 \pm 4.3$ & $16.6 \pm 3.2$ \\
\hline Dorzolamide $2 \% /$ timolol $0.5 \%$ & Brinzolamide $1 \% /$ timolol $0.5 \%$ & 2937 & $18.5 \pm 4.1$ & $16.5 \pm 3.2$ \\
\hline PGA/timolol $0.5 \% c$ & Brinzolamide I\%/timolol 0.5\% & 611 & $20.8 \pm 4.6$ & $17.8 \pm 4.2$ \\
\hline Brimonidine $0.2 \% /$ timolol $0.5 \%$ & Brinzolamide $1 \% /$ timolol $0.5 \%$ & 209 & $20.1 \pm 4.0$ & $17.4 \pm 3.3$ \\
\hline Brinzolamide $1 \%+$ timolol $0.5 \%$ & Brinzolamide $1 \% /$ timolol $0.5 \%$ & 252 & $18.4 \pm 3.4$ & $16.6 \pm 2.9$ \\
\hline Dorzolamide $2 \%$ + timolol $0.5 \%$ & Brinzolamide $1 \% /$ timolol $0.5 \%$ & 73 & $18.7 \pm 3.5$ & $16.5 \pm 2.9$ \\
\hline PGA + timolol $0.5 \%$ & Brinzolamide $1 \% /$ timolol $0.5 \%$ & 92 & $20.5 \pm 3.9$ & $17.8 \pm 3.1$ \\
\hline PGA + brinzolamide $1 \%$ & Brinzolamide $1 \% /$ timolol $0.5 \%+$ PGA & 150 & $20.9 \pm 4.8$ & $16.3 \pm 3.6$ \\
\hline PGA + dorzolamide $2 \%$ & Brinzolamide $1 \% /$ timolol $0.5 \%+$ PGA & 63 & $20.9 \pm 7.4$ & $16.4 \pm 5.3$ \\
\hline PGA + dorzolamide $2 \% /$ timolol $0.5 \%$ & Brinzolamide $1 \% /$ timolol $0.5 \%+$ PGA & 823 & $18.3 \pm 5.0$ & $16.4 \pm 3.9$ \\
\hline
\end{tabular}

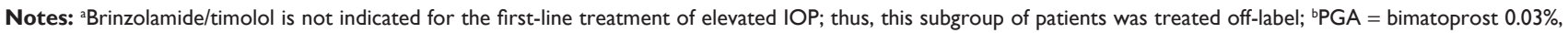
latanoprost $0.005 \%$, tafluprost $0.0015 \%$, or travoprost $0.004 \%$; ' Fixed-combination PGA/timolol $=$ latanoprost $0.005 \% /$ timolol $0.5 \%$, travoprost $0.004 \% /$ timolol $0.5 \%$, and bimatoprost $0.03 \% /$ timolol $0.5 \%$.

Abbreviations: IOP, intraocular pressure; PGA, prostaglandin analog. 


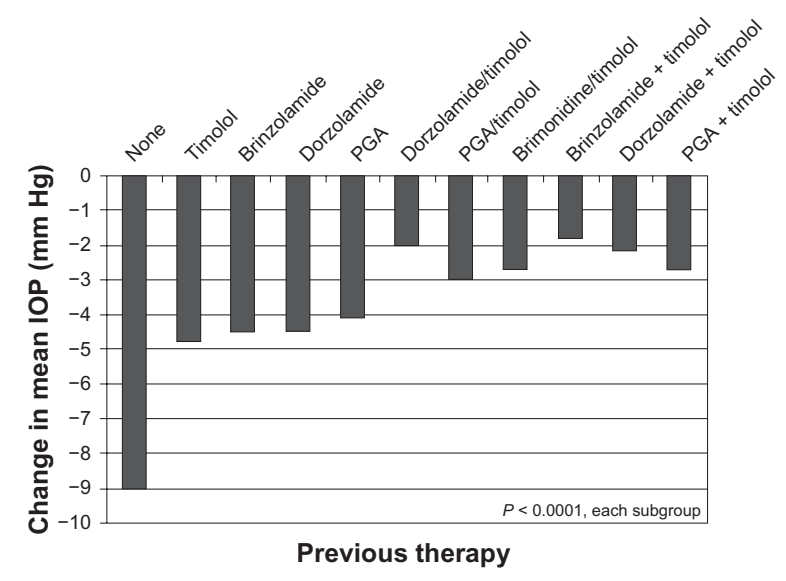

Figure IA Change in mean IOP from baseline to 4-6 weeks after transition to brinzolamide/timolol in patient subgroups with various previous therapies.

Abbreviations: IOP, intraocular pressure; PGA, prostaglandin analog.

of 14,025 investigators and $88.8 \%$ of 13,837 patients rating this regimen favorably (Figure 2). Investigators were more highly satisfied than patients with this regimen $(60.9 \%$ vs $53.5 \%$ excellent or very good).

\section{Patient preference}

Therapy preference data were evaluable for 13,346 patients. Of these patients, $75.9 \%$ preferred brinzolamide/timolol FC, whereas $8.6 \%$ favored their previous therapy, and $15.5 \%$ had no preference. Thus, of the patients with a therapy preference, brinzolamide/timolol was preferred over previous therapy at a ratio of almost 9:1.

\section{Comparison of non-PGA FC products with brinzolamide/timolol FC}

The most common previous therapy in this study was dorzolamide/timolol $(\mathrm{N}=2937)$, representing over $20 \%$ of

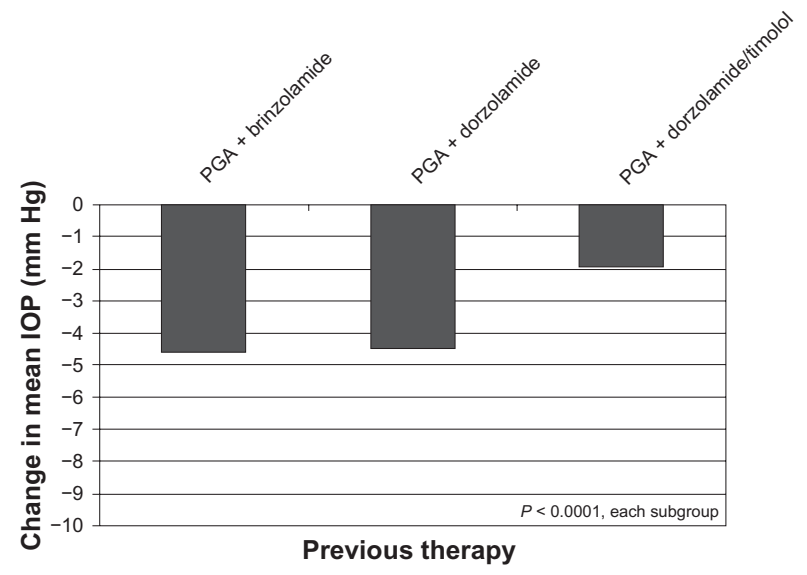

Figure IB Change in mean intraocular pressure from baseline to 4-6 weeks after transition to brinzolamide/timolol + a prostaglandin analog in patient subgroups with various previous therapies.

Abbreviations: IOP, intraocular pressure; PGA, prostaglandin analog. the total population. The majority of this subgroup of patients (56.7\%) transitioned to brinzolamide/timolol because of intolerance to dorzolamide/timolol (as judged by the treating physician; Table 3), a proportion that was substantially higher than intolerance to previous therapy cited in the overall population $(28.9 \%)$. When these patients were transitioned from dorzolamide/timolol to brinzolamide/timolol, mean IOP decreased by $10.8 \%$ from $18.5 \pm 4.1 \mathrm{~mm} \mathrm{Hg}$ to $16.5 \pm 3.2 \mathrm{~mm} \mathrm{Hg}(P<0.0001)$. These patients rated the tolerability of brinzolamide/timolol substantially higher than they did dorzolamide/timolol, with the positive responses of very good and good increasing from $29.2 \%$ with dorzolamide/ timolol to $88.9 \%$ with brinzolamide/timolol (Figure 3). Similar to the overall population, patients in this subgroup preferred brinzolamide/timolol over dorzolamide/timolol at a ratio of more than $9: 1$ ( $82.0 \%$ vs $8.8 \%$; Figure 4$)$. For the 209 patients who transitioned from FC brimonidine/timolol to brinzolamide/timolol, a reduction in mean IOP of $13.4 \%$ was observed $(20.1 \pm 4.0 \mathrm{~mm} \mathrm{Hg}$ vs $17.4 \pm 3.3 \mathrm{~mm} \mathrm{Hg}$; $P<0.0001)$. More patients rated brinzolamide/timolol favorably than they did brimonidine/timolol (86.5\% vs $32.1 \%$; Figure 5), and these patients preferred brinzolamide/timolol over brimonidine/timolol at a ratio of $11.5: 1$ (75.8\% vs 6.6\%; Figure 6).

\section{Comparison of PGA FC products to brinzolamide/timolol FC}

A total of 611 patients transitioned from FC PGA/timolol to brinzolamide/timolol, achieving a reduction in mean IOP of $14.4 \%(20.8 \pm 4.6 \mathrm{~mm} \mathrm{Hg}$ vs $17.8 \pm 4.2 \mathrm{~mm} \mathrm{Hg}$; $P<0.0001)$. More patients rated the tolerability of brinzolamide/timolol as very good or good than they did PGA/timolol (81.3\% vs 45.0\%), and 5.3-fold more of these patients preferred brinzolamide/timolol than they did PGA/ timolol (68.9\% vs $13.1 \%)$.

\section{Discussion}

In this large, noninterventional study examining the use of brinzolamide/timolol, patients were transitioned from their previous therapy to this product at their ophthalmologist's discretion, most frequently because of a failure to reach target IOP or because of intolerance to previous therapy. The transition to brinzolamide/timolol produced a $3.9 \mathrm{~mm} \mathrm{Hg}(18.8 \%)$ improvement in IOP after 4-6 weeks of use, for a mean IOP with brinzolamide/timolol of $16.8 \pm 3.6 \mathrm{~mm} \mathrm{Hg}$. A statistically significant reduction in mean IOP $(P<0.0001)$ was observed across all predefined subgroups, suggesting that brinzolamide/timolol was an effective second-line regimen, 


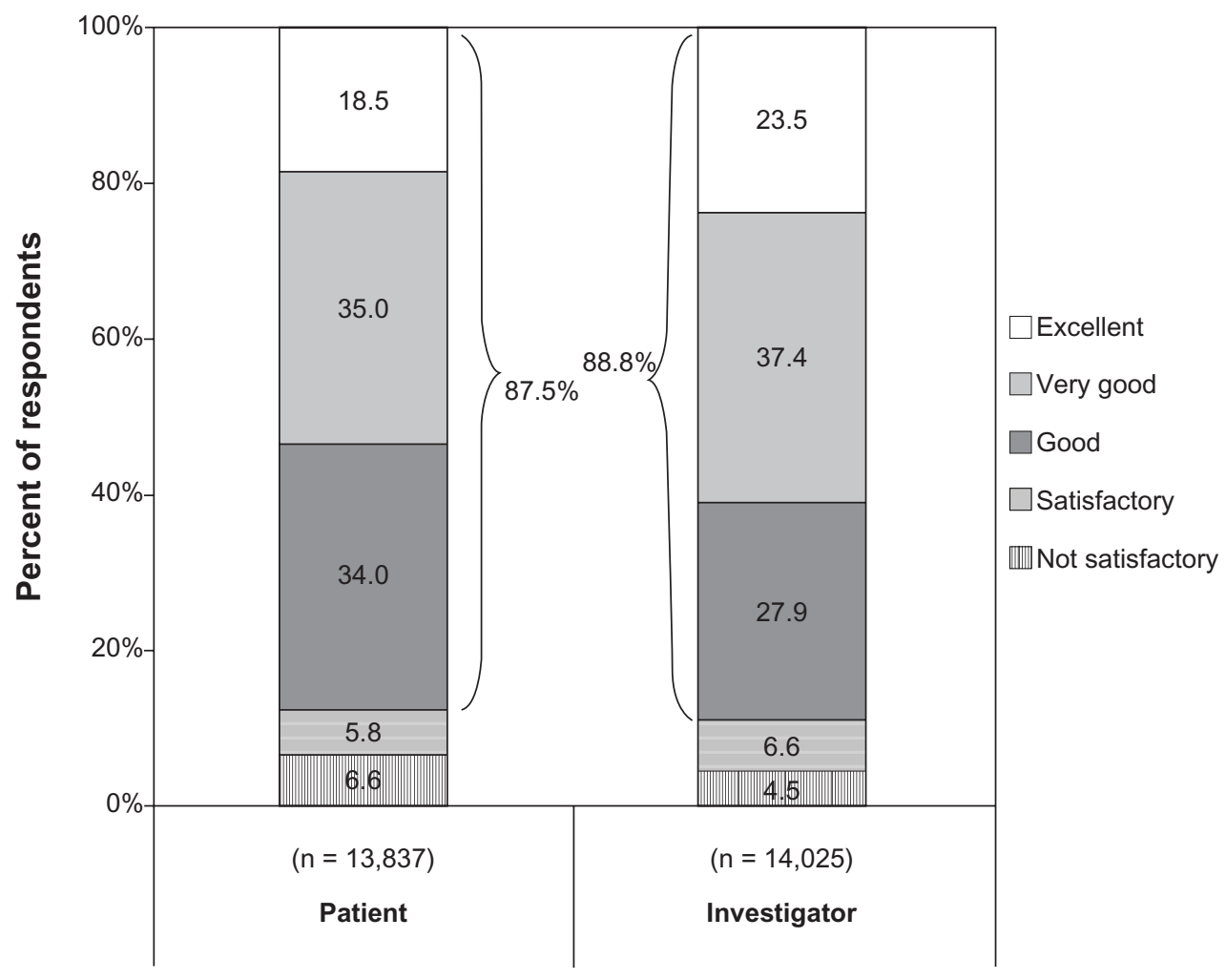

Figure 2 Patient- and investigator-assessed satisfaction with brinzolamide/timolol after 4-6 weeks of therapy.

producing additional reductions in IOP regardless of the type of previous therapy used.

A possible limitation of this type of noninterventional study is the lack of standardization of IOP measurement. However, a recent paper reported that three distinct methods of IOP measurement (dynamic contour, ocular response analyzer, and Goldmann applanation tonometry) produced clinically acceptable measurement repeatability and reproducibility, including acceptably low levels of interobserver variability. ${ }^{3}$ Thus, it appears likely that the lack of a standardized approach to IOP measurement had little effect on IOP outcome measures. In any case, the results reflect the

Table 3 Reasons for transition from dorzolamide/timolol to brinzolamide/timolol $(n=2937)$

\begin{tabular}{ll}
\hline $\begin{array}{l}\text { Reasons for transition } \\
\text { to brinzolamide/timolol } \\
\text { (multiple answers may apply) }\end{array}$ & $\begin{array}{l}\text { Percentage } \\
\text { of patients }\end{array}$ \\
\hline $\begin{array}{l}\text { Intolerance to previous therapy } \\
\text { Cost reduction }\end{array}$ & 56.7 \\
$\begin{array}{l}\text { Insufficient intraocular pressure lowering } \\
\text { under previous therapy }\end{array}$ & 27.7 \\
$\begin{array}{l}\text { Insufficient patient compliance with } \\
\text { previous therapy }\end{array}$ & 23.7 \\
Progressive disease & 8.6 \\
Vascular aspects & \\
\hline
\end{tabular}

real-world data used by ophthalmologists to make treatment decisions.

The tolerability of brinzolamide/timolol was overwhelmingly positive, with nearly $90 \%$ of patients judging its tolerability to be either good or very good. This was in contrast with previous therapy, for which just over half of the patients rated their previous IOP-lowering medication positively. Although this was not surprising, given that nearly $30 \%$ of patients transitioned to brinzolamide/timolol due to intolerance to their previous therapy, it indicated that this FC product is a favorable choice in these circumstances. Supporting this conclusion is the high patient satisfaction with brinzolamide/timolol reported in the current study (93.4\% satisfied) and the good safety profile of brinzolamide/ timolol, producing ocular adverse events such as blurred vision, eye pain or irritation, and foreign body sensation in $<10 \%$ of patients. ${ }^{1}$ The fact that patients reported a preference for their new regimen over their previous regimen at a ratio of nearly 9:1 further substantiates this claim. However, it should be noted that because of the limited duration of brinzolamide/timolol therapy prior to patient assessment (4-6 weeks), any long-term side effects would not have yet developed, resulting in the possibility of an artificially favorable tolerability profile of brinzolamide/timolol compared with previous therapy. 


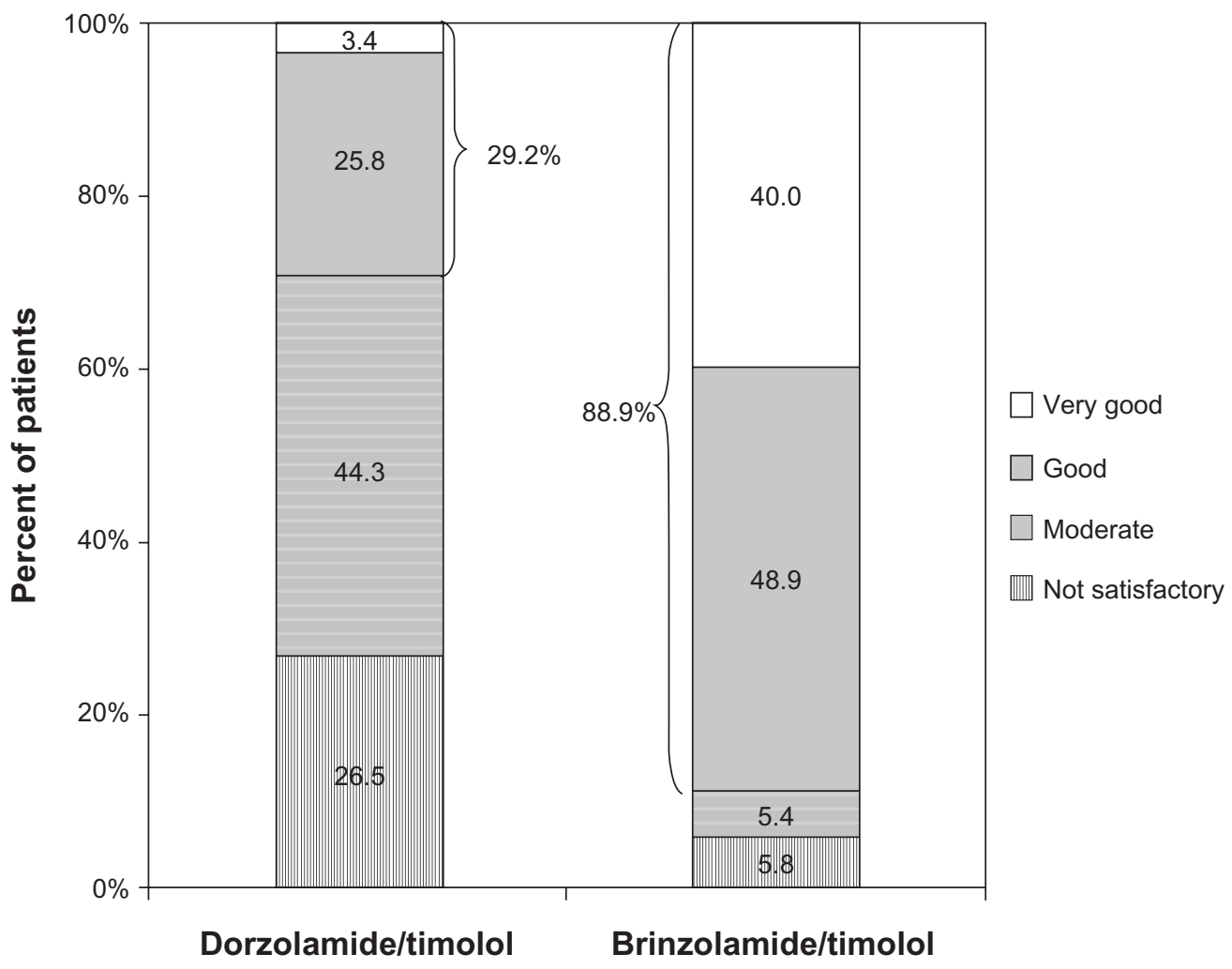

Figure 3 Patient-reported tolerability of dorzolamide/timolol vs brinzolamide/timolol $(n=2729)$.

The results of this study should be considered in light of two phenomena: the Hawthorne effect and regression to the mean. The Hawthorne effect describes a phenomenon in which study results show improvement simply because a measure is being assessed rather than because an intervention is having a true positive effect. ${ }^{4}$ In the current study, it is feasible that changing a patient's medication may have produced improved outcomes regardless of which medication

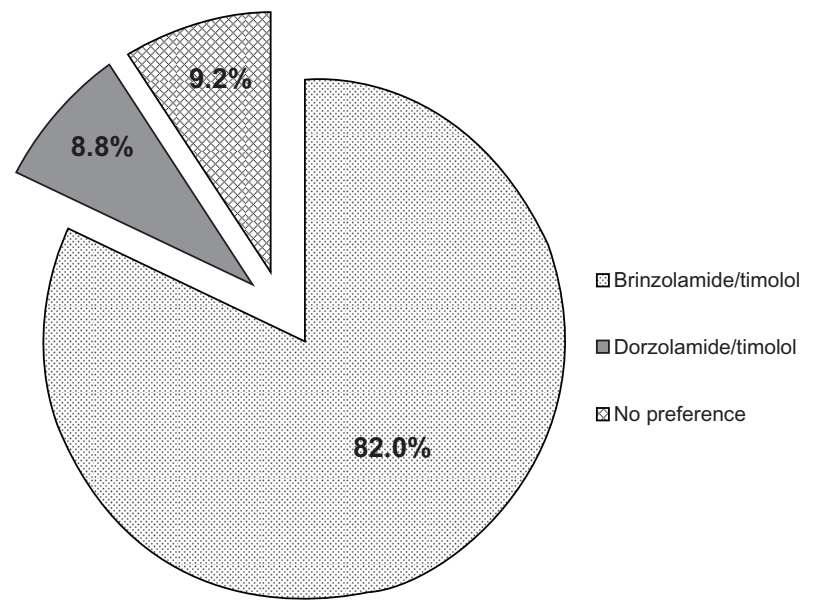

Figure 4 Patient preference between dorzolamide/timolol and brinzolamide/timolol 4-6 weeks after transition to brinzolamide/timolol $(n=2819)$. was prescribed. Although it is plausible that this may have impacted the patient assessment results of tolerability and drug preference, it is less likely to have impacted an objective measure like IOP. The second phenomenon of regression to the mean suggests that the favorable results of brinzolamide/ timolol may lessen over time, particularly since the follow-up was short. To determine whether this is indeed the case, this medication would have to be examined in a long-term study (ie, having at least 1 year of follow-up).

This observational study also provided an opportunity to compare the real-world use of two similar products, the CAIcontaining FC dorzolamide/timolol and brinzolamide/ timolol. Nearly 3000 enrolled patients transitioned from the dorzolamide combination product to brinzolamide/timolol, with more than half of them doing so because of their intolerance to their previous therapy, according to their treating physician. This was substantially higher than the $28.9 \%$ of patients from the total study population who made a change due to intolerance. In fact, dorzolamide/timolol has a challenging safety profile, producing dysgeusia and ocular burning and/or stinging in up to $30 \%$ of patients. ${ }^{5}$ In the current study, less than one-third of patients taking dorzolamide/ timolol rated its tolerability positively, and more than onequarter judged it to be unsatisfactory. After transitioning to 


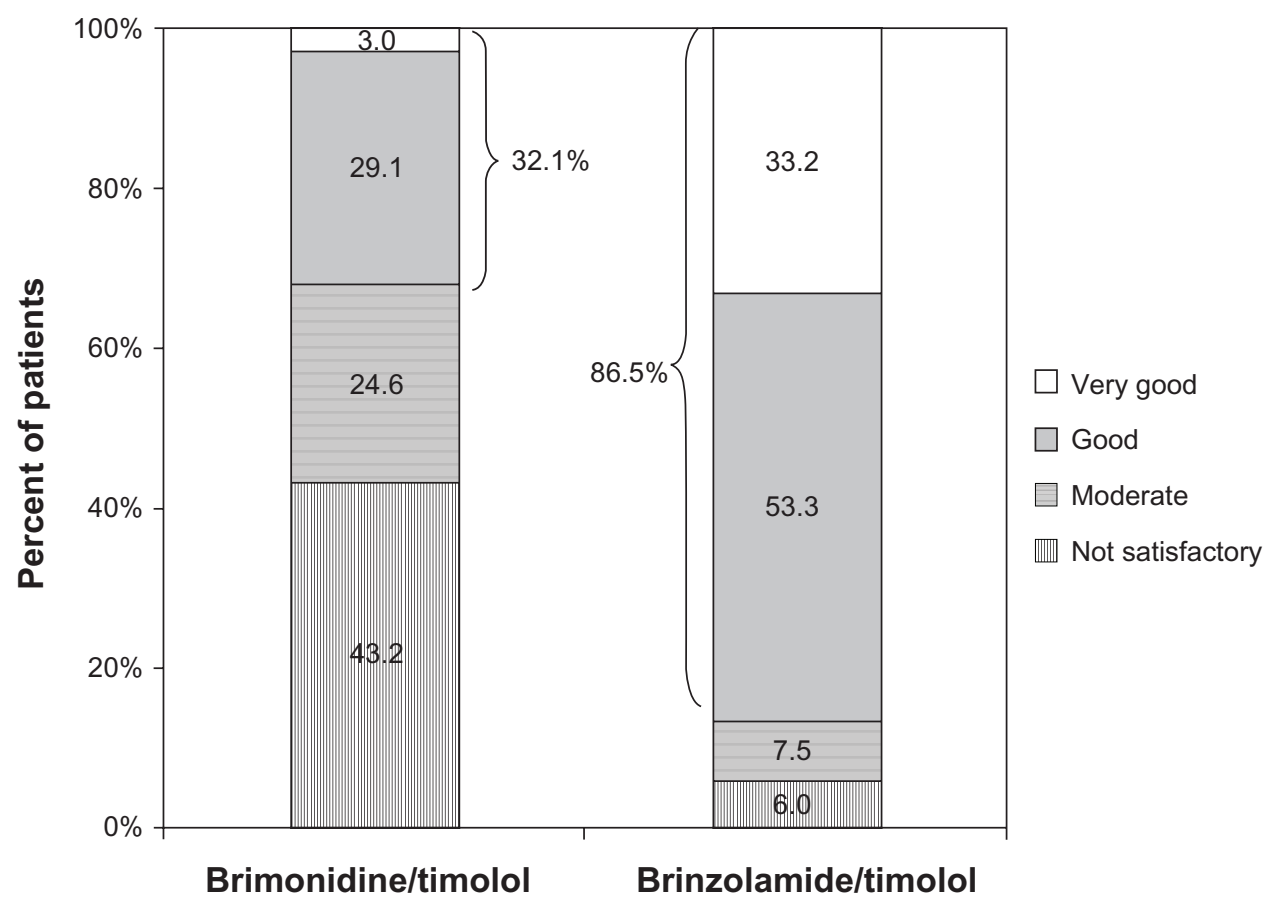

Figure 5 Patient-reported tolerability of brimonidine/timolol vs brinzolamide/timolol $(n=209)$.

brinzolamide/timolol, the vast majority of these patients rated the tolerability of their new regimen positively, indicating that they found brinzolamide/timolol to be more comfortable than dorzolamide/timolol. This hypothesis was supported by results from a clinical trial designed to directly compare the comfort of these two products. ${ }^{6}$ In this randomized, double-masked study, the brinzolamide/timolol group had a significantly lower mean ocular discomfort score $(P=0.0003)$ and a greater incidence of patients reporting no ocular discomfort after 1 week of therapy than the dorzolamide/timolol group ( $49 \%$ vs $15 \%, P=0.0004)$. The apparent increase in comfort of brinzolamide/timolol therapy may be due to its

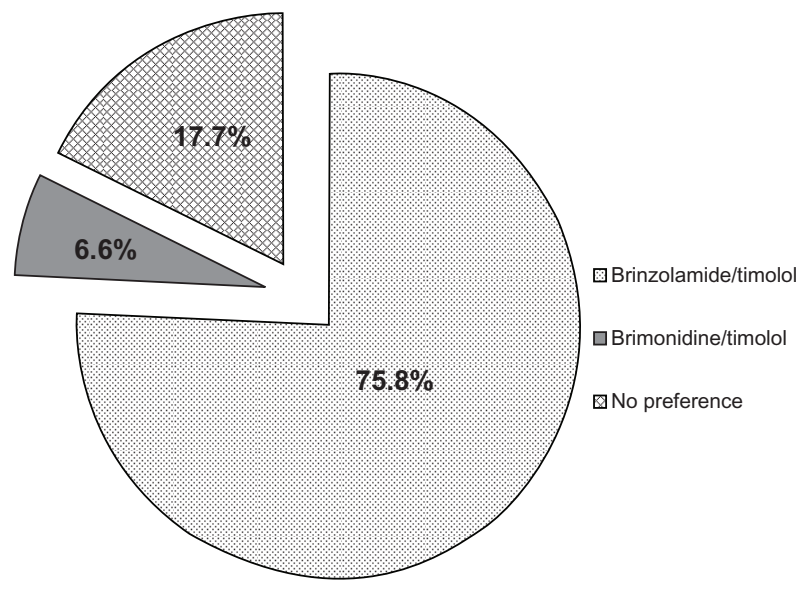

Figure 6 Patient preference between brimonidine/timolol and brinzolamide/timolol 4-6 weeks after transition to brinzolamide/timolol $(n=209)$. near physiologic $\mathrm{pH}$ of $7.2{ }^{6}{ }^{6}$ in contrast, dorzolamide/timolol has an acidic $\mathrm{pH}$ of 5.65. ${ }^{5}$ Again, patient preference data from the current study support the favorable tolerability of brinzolamide/timolol, which was preferred more than 9:1 over dorzolamide/timolol.

Despite the similar mechanisms of action of these two CAI-containing FC treatments, a modest but statistically significant reduction in IOP was observed in the current study on transitioning to brinzolamide/timolol therapy. Interestingly, a randomized noninferiority trial of 437 patients comparing these two combinations reported similar IOP reductions with both products. ${ }^{7}$ However, the reduction in IOP from the current study can potentially be explained if patients had better adherence to the brinzolamide/timolol regimen. It is reasonable to suggest that patients may be more inclined to comply with an agent with a favorable tolerability profile than with one that causes discomfort. In fact, a positive relationship between tolerability and patient adherence has been suggested in several glaucoma publications. ${ }^{8-11}$ This hypothesis, however, remains to be tested in a rigorous manner.

Brinzolamide/timolol also compared favorably against other FC products, specifically PGA/timolol products and $\alpha-2$ agonist-containing brimonidine/timolol. Despite the relatively small number of patients included in these subsets ( $\mathrm{n}=611$ and $\mathrm{n}=209$, respectively), both groups demonstrated significant improvements in IOP after transition to brinzolamide/timolol, and most patients in both groups found 
brinzolamide/timolol to be more tolerable and preferred over their previous therapy. This preference imbalance was particularly evident in the brimonidine/timolol group, where patient preference was 11.5:1 for brinzolamide/timolol over previous therapy.

Although the nonrandomized, open-label nature of a noninterventional study limits the type of conclusions that can be drawn from its results, it does have some advantages over data obtained from a clinical trial. That is, an observational trial usually involves a much larger patient population, making it more likely to obtain an accurate picture of the true effect of a drug on its patient population. Furthermore, unlike a highly regulated clinical trial with a restricted patient population and strictly defined usage, a noninterventional study can accurately reveal the efficacy and safety of a drug as it is truly used in clinical practice.

In the real-world setting of the current study, brinzolamide $1 \% /$ timolol $0.5 \%$ FC produced better IOP control than all previous therapies analyzed and demonstrated favorable tolerability, resulting in a strong patient preference for brinzolamide/timolol over other therapies. These data, collected from the daily practice of more than 1100 ophthalmologists across Germany, demonstrated that brinzolamide/ timolol was an effective second-line choice for the treatment of patients transitioning to a new IOP-lowering regimen.

\section{Acknowledgment}

Jennifer Klem PhD provided medical writing assistance, which was funded by Alcon Laboratories, Inc.

\section{Disclosure}

The authors report no conflicts of interest in this work.

\section{References}

1. Summary of Product Characteristics (SPC) Azarga ${ }^{\circledR}$. Alcon Pharma, 2008.

2. Bundesinstitut fur Arzneimittel und Medizinprodukte website. Arzneimittelgesetz (AMG). http://www.bfarm.de/EN/BfArM/BfArM Service/AMG_en/amg-node-en.html. Accessed September 24, 2010.

3. Sullivan-Mee M, Gerhardt G, Halverson KD, Qualls C. Repeatability and reproducibility for intraocular pressure measurement by dynamic contour, ocular response analyzer, and goldmann applanation tonometry. J Glaucoma. 2009;18(9):666-673.

4. Mayo E. The Social Problems of an Industrial Civilisation. London: Routledge; 1949.

5. COSOPT [package insert]. Whitehouse Station, NJ: Merck \& Co., Inc.; 2006.

6. Vold SD, Evans RM, Stewart RH, et al; for the Brinzolamide/Timolol Comfort Study Group. A one-week comfort study of BID-dosed brinzolamide $1 \% /$ timolol $0.5 \%$ ophthalmic suspension fixed combination compared to BID-dosed dorzolamide $2 \% /$ timolol $0.5 \%$ ophthalmic solution in patients with open-angle glaucoma or ocular hypertension. J Ocul Pharm Ther. 2008;24(6):601-605.

7. Manni G, Denis $P$, Chew $P$, et al. The safety and efficacy of brinzolamide $1 \% /$ timolol $0.5 \%$ fixed combination versus dorzolamide $2 \% /$ timolol $0.5 \%$ in patients with open-angle glaucoma or ocular hypertension. J Glaucoma. 2009;18(4):293-300.

8. Silva LR, de Paula JS, Rocha EM, Rodrigues Mde L. Factors related to glaucoma treatment compliance: patients' opinions from a university hospital. Arq Bras Oftalmol. 2010;73(2):116-119.

9. Chan K, Testa M, McCluskey P. Ocular comfort of combination glaucoma therapies: brimonidine $0.2 \% /$ timolol $0.5 \%$ compared with dorzolamide 2\%/timolol 0.5\%. J Ocul Pharmacol Ther. 2007;23(4): 372-376.

10. Tsai JC, McClure CA, Ramos SE, et al. Compliance barriers in glaucoma: a systematic classification. J Glaucoma. 2003;12(5):393-398.

11. Barnebey H, Kwok SY. Patients' acceptance of a switch from dorzolamide to brinzolamide for the treatment of glaucoma in a clinical practice setting. Clin Ther. 2000;22(10):1204-1212.
Clinical Ophthalmology

\section{Publish your work in this journal}

Clinical Ophthalmology is an international, peer-reviewed journal covering all subspecialties within ophthalmology. Key topics include: Optometry; Visual science; Pharmacology and drug therapy in eye diseases; Basic Sciences; Primary and Secondary eye care; Patient Safety and Quality of Care Improvements. This journal is indexed on Submit your manuscript here: http://www.dovepress.com/clinical-ophthalmology-journal

\section{Dovepress}

PubMed Central and CAS, and is the official journal of The Society of Clinical Ophthalmology (SCO). The manuscript management system is completely online and includes a very quick and fair peer-review system, which is all easy to use. Visit http://www.dovepress.com/ testimonials.php to read real quotes from published authors. 